\title{
Strong Differential Photoion Circular Dichroism in Strong-Field Ionization of Chiral Molecules
}

\author{
K. Fehre $\odot,{ }^{1, *}$ S. Eckart $\odot,{ }^{1}$ M. Kunitski $\odot,{ }^{1}$ C. Janke, ${ }^{1}$ D. Trabert, ${ }^{1}$ M. Hofmann, ${ }^{1}$ J. Rist $\odot,{ }^{1}$ M. Weller, ${ }^{1}$ A. Hartung, ${ }^{1}$ \\ L. Ph. H. Schmidt, ${ }^{1}$ T. Jahnke, ${ }^{1}$ H. Braun ${ }^{2}{ }^{2}{ }^{T}$. Baumert ${ }^{2}{ }^{2}$ J. Stohner, ${ }^{3}$ Ph. V. Demekhin, ${ }^{2}$ \\ M. S. Schöffler, ${ }^{1, \dagger}$ and R. Dörner( ${ }^{1,+}$ \\ ${ }^{1}$ Institut für Kernphysik, Goethe-Universität, Frankfurt Max-von-Laue-Straße 1, 60438 Frankfurt am Main, Germany \\ ${ }^{2}$ Institut für Physik und CINSaT, Universität Kassel, Heinrich-Plett-Straße 40, 34132 Kassel, Germany \\ ${ }^{3}$ ZHAW Zurich University for Applied Sciences, Department N, Campus Reidbach, \\ Research Group Physical Chemistry Einsiedlerstrasse 31, 8820 Wädenswil, Switzerland
}

(Received 20 July 2020; accepted 15 January 2021; published 25 February 2021)

\begin{abstract}
We investigate the differential ionization probability of chiral molecules in the strong-field regime as a function of the helicity of the incident light. To this end, we analyze the fourfold ionization of bromochlorofluoromethane $(\mathrm{CHBrClF})$ with subsequent fragmentation into four charged fragments and different dissociation channels of the singly ionized methyloxirane. By resolving for the molecular orientation, we show that the photoion circular dichroism signal strength is increased by 2 orders of magnitude.
\end{abstract}

DOI: 10.1103/PhysRevLett.126.083201

Circular dichroism (CD) effects depict the difference in the absorption strength between left- and right-handed circularly polarized (LCP and RCP, respectively) light occurring for the two enantiomers of a chiral substance. $\mathrm{CD}$ effects have received great attention for many years with a variety of possible applications in, for example, the fields of absorption spectroscopy [1] or fluorescence spectroscopy [2]. Since the probability of absorption is directly linked to the probability of photoionization [3], an occurring $\mathrm{CD}$ can lead to observable differences in the absolute ion yield [4]. CD effects are usually very small; their theoretical description relies on light-matter interaction beyond the electrical dipole approximation (see Supplemental Material [5]), which is a general feature for all scalar observables connected to the scope of CD [9]. Thus, one promising route to enhance the visibility of $\mathrm{CD}$ effects is to examine vectorial observables such as electron emission angles (electron momenta) rather than rates or yields. One prime example showing the strength of such an approach is the photoelectron circular dichroism (PECD), which is the normalized change of photoelectron angular emission distributions from chiral molecules upon inversion of the light helicity. The PECD displays signal strengths increased by orders of magnitude [10-13] as compared to the emission angle-integrated $\mathrm{CD}$. The

Published by the American Physical Society under the terms of the Creative Commons Attribution 4.0 International license. Further distribution of this work must maintain attribution to the author(s) and the published article's title, journal citation, and DOI. ionization probability of molecules can strongly depend on the orientation of the molecule with respect to the laser, which has been shown experimentally [14] (see Fig. 2) and can now be routinely modeled by standard quantum chemistry program packages [15]. The chiral effect discussed in this Letter, however, is the influence of the helicity of the light on the ionization probability that is resolved on the fragmentation direction of the molecule with respect to the light propagation direction.

While typical enhancements of the photoion circular dichroism (PICD) for a fixed molecular orientation are rather small for single photon ionization [16], a recent experiment fosters the hope for much bigger effects in the strong-field case [17]. In this experiment, an achiral molecule was excited and its chiral fragmentation detected. While the light's helicity had only a very slight influence on the total ion yield of an enantiomer, the differential PICD led to a signal amplification of up to 2 orders of magnitude. However, the observed changes in the differential ionization probability were inextricably linked to the selective generation of one of the two enantiomers. Hence, the observation of a strong differential PICD employing a real chiral molecule is still pending.

The experiments reported here have been performed using the ion arm of a COLTRIMS (cold target recoil ion momentum Spectroscopy) [18] reaction microscope as described in previous publications $[13,17,19]$. Two independent experiments were performed using bromochlorofluoromethane $(\mathrm{CHBrClF})$ and methyloxirane $\left(\mathrm{C}_{3} \mathrm{H}_{6} \mathrm{O}\right)$. The ionization of the $\mathrm{CHBrClF} /$ methyloxirane molecules was induced by focusing short, intense laser pulses $[f=60 \mathrm{~mm}, 40 \mathrm{fs}$, beam diameter 8.2/4 mm (FWHM), 
central wave length $800 \mathrm{~nm}, 2.5 \mathrm{~W} / 0.3 \mathrm{~W}$, generated by a Ti:sapphire regenerative amplifier (KMLabs Wyvern 500), resulting in a focal intensity of $2.5 \times 10^{15} \mathrm{~W} / \mathrm{cm}^{2} /$ $6.9 \times 10^{13} \mathrm{~W} / \mathrm{cm}^{2}$ onto supersonic gas jets of either a racemic mixture of $\mathrm{CHBrClF}$ or enantiopure $\mathrm{C}_{3} \mathrm{H}_{6} \mathrm{O}$. Highefficiency microchannel plates were used [20]. With the ionization potential of the HOMO $(10.25 \mathrm{eV})$ and HOMO$1(11.23 \mathrm{eV})$ [21] (methyloxirane), the above given parameters result in the Keldysh parameters of $\gamma=1.115$ and $\gamma=1.167$. During the data acquisition, a motorized stage switched the helicity of the light every 3 minutes to ensure identical experimental conditions for the measurements with left- and right-handed polarized light. Generation of LCP and RCP light was done by using a set of a half- and a quarter-wave plates. The angles of the plates were optimized by minimizing the ionization yield of argon. By examining the three-dimensional momentum distribution of the photoelectrons measured in coincidence [22], we estimate that the field ratio $\left(\boldsymbol{E}_{1} / \boldsymbol{E}_{2}\right)>0.95$. A systematic error due to imperfect circular polarization thus reduces the strength of the chiral signal by a few percent of the actual signal. This error is therefore smaller than the specified statistical errors. The gas jet was produced by expanding $\mathrm{CHBrClF} /$ methyloxirane vapor (vapor pressure at room temperature is approximately $600 / 588 \mathrm{mbar}$, respectively) through a nozzle of $30 \mu \mathrm{m}$ diameter into vacuum and differentially pumped or shaped by three stages with (adjustable) apertures.

In the present experiment we examine the four-body fragmentation of $\mathrm{CHBrClF} \rightarrow \mathrm{CH}^{+}+\mathrm{Br}^{+}+\mathrm{Cl}^{+}+\mathrm{F}^{+}+4 e^{-}$. We ionize molecules from a racemic sample and determine the handedness of each individual molecule from the triple product of three of the four momentum vectors of the fragments measured in coincidence [23]. For example, $\overrightarrow{p_{\mathrm{F}}} \cdot\left(\overrightarrow{p_{\mathrm{Cl}}} \times \overrightarrow{p_{\mathrm{Br}}}\right) /\left(\left|\overrightarrow{p_{\mathrm{F}}}\right| \cdot\left|\overrightarrow{p_{\mathrm{Cl}}} \times \overrightarrow{p_{\mathrm{Br}}}\right|\right)<0 \quad(>0)$ indicates the ionization of the $S(R)$ enantiomer. $\overrightarrow{p_{F}}, \overrightarrow{p_{\mathrm{Cl}}}$, and $\overrightarrow{p_{\mathrm{Br}}}$ are the measured momentum vectors of the singly charged fluorine, chlorine, and bromine ions.

A chemical synthesis of chiral molecules without specific asymmetric synthesis aspects of enantioseparation results in a perfect racemate; the ratio between $R$ and $S$ enantiomers is $1: 1$. Therefore, both enantiomers are subject to identical experimental conditions and provide a perfect cross-check for the measured circular dichroism in the ion yield. The measurements with the two light helicities show a normalized difference in PICD = $\left[\left(R_{\mathrm{RCP}}-R_{\mathrm{LCP}}\right) /\left(R_{\mathrm{RCP}}+R_{\mathrm{LCP}}\right)\right]=(0.094 \pm 0.046) \%$ the in the fourfold fragmentation of $\mathrm{CHBrClF}$ for both enantiomers (integrated over all molecular orientations). $R_{\mathrm{LCP}}$ $\left(R_{\mathrm{RCP}} R\right)$ indicate the measured count rate of the measured four-body fragmentation channel of the enantiomer for LCP (RCP). In order to compensate for slight differences in the measuring time, the totals of the count rates for $R_{\mathrm{RCP}}$ and $S_{\mathrm{RCP}}\left(R_{\mathrm{LCP}}\right.$ and $\left.S_{\mathrm{LCP}}\right)$ were normalized to one. Performing the data analysis on the mirror image molecule

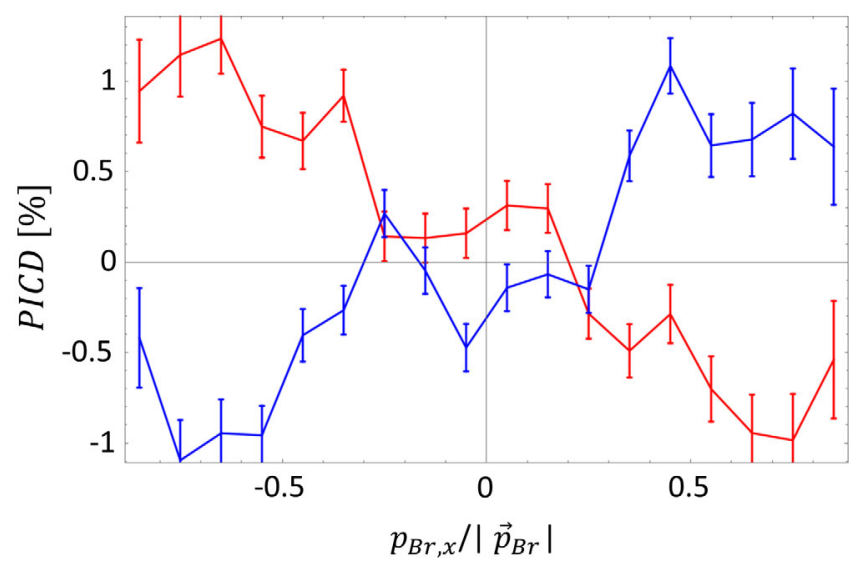

FIG. 1. Differential PICD as normalized difference between the enantiomers for $\mathrm{CHBrClF}$ as function of the angle between the bromine ion momentum and the direction of light propagation. The red curve reflects the measurement results for LCP, the blue for RCP. The error bars indicate the statistical error.

yielded the exact same result $[(-0.094 \pm 0.046) \%]$ with the expected change of sign.

Our fourfold coincidence measurement of ion momenta allows us to inspect the data on different levels of detail. A more differential than the ion count rate and yet very basic vectorial observable is the PICD as a function of the direction of one of the ion momenta with respect to the light propagation axis. This is the ion equivalent to angular resolved PECD.

We choose the $\mathrm{Br}^{+}$ion for that purpose and plot in Fig. 1 the PICD, defined as $\operatorname{PICD}\left(\overrightarrow{p_{\mathrm{Br}}}\right)=\left\{\left[R_{\mathrm{LCP}}\left(\overrightarrow{p_{\mathrm{Br}}}\right)-S_{\mathrm{LCP}}\left(\overrightarrow{p_{\mathrm{Br}}}\right)\right] /\right.$ $\left.\left[R_{\mathrm{LCP}}\left(\overrightarrow{p_{\mathrm{Br}}}\right)+S_{\mathrm{LCP}}\left(\overrightarrow{p_{\mathrm{Br}}}\right)\right]\right\}$, as a function of the cosine of the angle between the $\mathrm{Br}^{+}$momentum and the light propagation axis ( $x$ direction). We find a PICD of up to $1 \%$ for a $\mathrm{Br}^{+}$ion emission along the light propagation inverting its sign for the opposite $\mathrm{Br}^{+}$emission direction or light helicity.

Note that we use the normalized difference between the enantiomers to calculate the PICD, as this representation shows the forward-backward asymmetry, as it is known from experiments on the photoelectron circular dichroism $[11,12,24]$. However, for (partially) oriented molecules, the differential PICD shows a different pattern comparing the two light helicities or the two enantiomers (Fig. S2 in Supplemental Material [5]). A comparable observation has already been made for the photoelectron circular dichroism when examining it with elliptically polarized light [19]. While the investigation of the influence of light helicity on the differential ionization probability changes only the chiral interaction partner, in the comparison of the enantiomers also the differences in the coordinate system is taken into account. A further discussion is found in the Supplemental Material [5].

As the next step we inspect the fully differential PICD incorporating all ionic fragments. For this purpose, we 


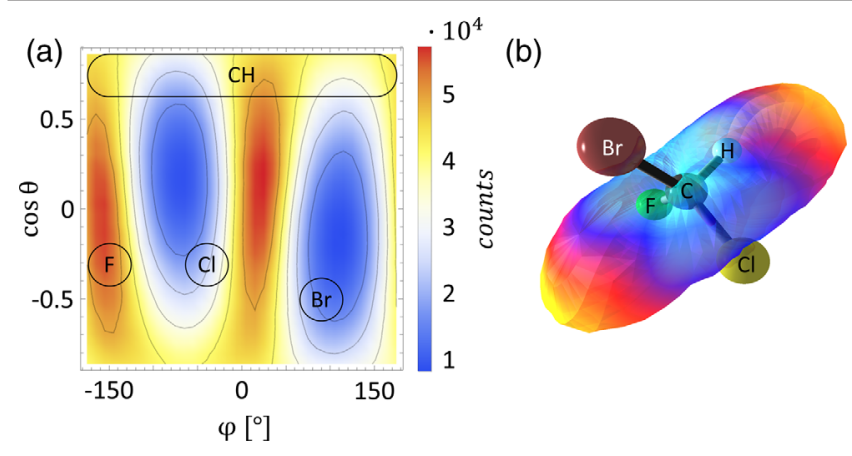

FIG. 2. Differential ionization probability for $R \mathrm{CHBrClF}$ and LCP. (a) Each point in the graph shows the number of measured events for a direction of light propagation in spherical coordinates in the molecular system. The position of the molecule in the selected molecular system is indicated by the position of the fragments' momenta. (b) The colored sphere represents the differential count rate in the molecular system. The count rate is represented by the distance from the $\mathrm{C}$ atom and the color.

switch to a molecular coordinate frame. In this system, the momentum of the $\mathrm{CH}^{+}$fragment defines the $z^{\prime}$ axis, the $\mathrm{Br}^{+}$fragment emission direction defines the $z^{\prime} y^{\prime}$ plane pointing in positive $y^{\prime}$ direction. All ionic fragment emission directions as well as the light propagation direction are transformed into the molecular system. The result, converted into the angles of the spherical coordinates, is shown in Fig. 2.

Each point in the map in Fig. 2(a) corresponds to a distinct light propagation direction in the molecular frame.

Now we can turn to the investigation of the differential PICD. Figure 3 shows the corresponding normalized difference between the ionization with LCP and RCP of $\mathrm{CHBrClF}$ and the aforementioned fourfold fragmentation channel. PICD values of up to $10 \%$ are observable and thus show signal strength that is 2 orders of magnitude larger than the CD in the ion yield. The PICD patterns show in first approximation a change of sign in the PICD for molecular orientations in which the light in the molecular system impinges on the molecule from opposite directions. This symmetry is expected for effects that can be described in the context of the electrical dipole approximation as in this context, a reversal of the light direction in the molecular system is equivalent to a reversal of the light helicity. In the presented PICD maps, molecular orientations corresponding to the antisymmetry are linked by a change of sign in $\cos \theta$ and a translation of $\pm 180^{\circ}$ in $\varphi$. For small values of $\cos \theta$ there is a deviation of this antisymmetry in the PICD pattern. This deviation suggests that the signal amplification of the differential PICD cannot be explained solely in terms of the electrical dipole approximation. Note that within the electrical dipole approximation experimental errors cannot violate the expected antisymmetry. Experimental errors such as distorted spectrometer potentials or sensitivity inhomogeneities on the detector do not influence the PICD. As we present in Fig. 3

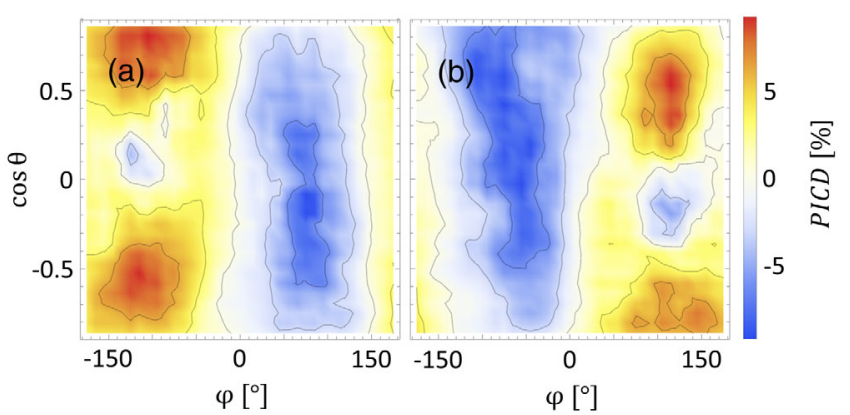

FIG. 3. Differential PICD in the four-body fragmentation of $\mathrm{CHBrClF}$. (a) $\operatorname{PICD}[\varphi, \cos (\theta)]=100 *\left\{\left(S_{\mathrm{RCP}}[\varphi, \cos (\theta)]-\right.\right.$ $\left.\left.S_{\mathrm{LCP}}[\varphi, \cos (\theta)]\right) /\left(S_{\mathrm{RCP}}[\varphi, \cos (\theta)]+S_{\mathrm{LCP}}[\varphi, \cos (\theta)]\right)\right\}$ for the $S$ enantiomer. (b) PICD as in (a) for the $R$ enantiomer. For small values of $\cos \theta$ there is a deviation from the antisymmetry in the PICD pattern.

a comparison between identical molecular orientations, these errors cancel out in the normalized difference. Any imperfection in the polarization state can influence the PICD value, but as all molecular orientations are subject to the identical light field, in the electric dipole approximation the PICD shows the antisymmetry.

As the definition of a joint molecular system is not possible for the two enantiomers of a chiral molecule, it is initially not clear which points on the two PICD maps are to be compared. At first glance, the two PICD patterns of the enantiomers are connected by a mirror operation (mirror plane located between the panels). A closer look suggests a different symmetry relationship. The negative contribution in Fig. 3(a) at $\varphi=-100^{\circ}$ is at $\cos \theta=+0.2$, and in Fig. 3(b) it is at about $\varphi=100^{\circ}$ and $\cos \theta=-0.2$. Therefore, a point inversion at $\varphi=0$ and $\cos \theta=0$ seems to be a more suitable symmetry operation to connect the PICD patterns of the two enantiomers.

Fourfold ionization as in the case of $\mathrm{CHBrClF}$ is a rare event. In most interactions with a laser pulse, only one electron is emitted. Thus, for analytical purposes it is desirable to study PICD also in single ionization events. In many such cases the molecule still fragments fast in a neutral and a charged fragment, which makes PICD as a function of the fragment emission direction accessible [25].

Note that the COLTRIMS technique used in the presented experiment separates the time of flight direction and the forward or backward direction with respect to the light propagation direction. We inspect the PICD in a twodimensional representation showing the position of impact of a particle on the detector versus the time of flight. Such a histogram is shown in Fig. 4 employing methyloxirane as a target. However, the differential PICD, in which only one molecular axis is resolved, is already accessible in a timeof-flight measurement, if the laser propagates not perpendicularly to the extraction direction of the time-of-flight spectrometer. 


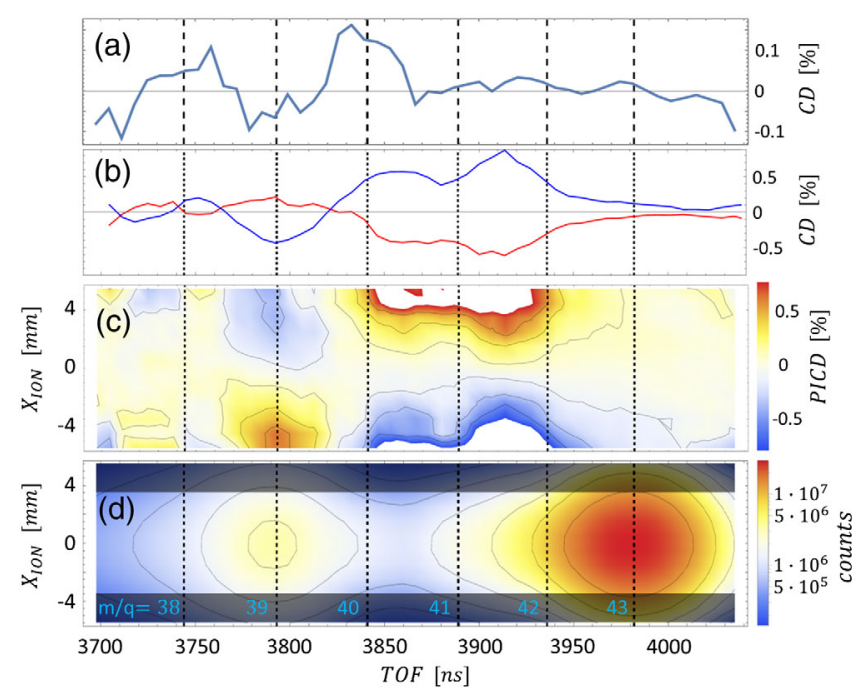

FIG. 4. Circular dichroism in the single ionization of methyloxirane. (a) Circular dichroism in the ion yield calculated by $\mathrm{CD}(\mathrm{TOF})=50\left\{\left[R_{\mathrm{LCP}}(\mathrm{TOF})-S_{\mathrm{LCP}}(\mathrm{TOF})\right] /\right.$ $\left.\left[R_{\mathrm{LCP}}(\mathrm{TOF})+S_{\mathrm{LCP}}(, \mathrm{TOF})\right]\right\}-50\left\{\left[R_{\mathrm{RCP}}(\mathrm{TOF})-S_{\mathrm{RCP}}(\mathrm{TOF})\right] /\right.$ $\left.\left[R_{\mathrm{RCP}}(\mathrm{TOF})+S_{\mathrm{RCP}}(, \mathrm{TOF})\right]\right\}-0.4$. Different measuring times and the smallest errors directly influence the $\mathrm{CD}$. The applied small correction cannot be determined from the available data. A detailed discussion of the errors can be found in the Supplemental Material [5]. (b) As in (a) with the additional condition that the location of the ion hit on the detector in the light propagation direction is larger (smaller) than $3.5 \mathrm{~mm}(-3.5 \mathrm{~mm})$, represented by the red (blue) line. With this condition, a subset of molecular orientations is selected. (c) $\operatorname{PICD}\left(X_{\mathrm{ION}}, \mathrm{TOF}\right)=$ $50\left\{\left[R_{\mathrm{LCP}}\left(X_{\mathrm{ION}}, \mathrm{TOF}\right)-S_{\mathrm{LCP}}\left(X_{\mathrm{ION}}, \mathrm{TOF}\right)\right] /\left[R_{\mathrm{LCP}}\left(X_{\mathrm{ION}}, \mathrm{TOF}\right)+\right.\right.$ $\left.\left.S_{\mathrm{LCP}}\left(X_{\mathrm{ION}}, \mathrm{TOF}\right)\right]\right\}-50\left\{\left[R_{\mathrm{RCP}}\left(X_{\mathrm{ION}}, \mathrm{TOF}\right)-S_{\mathrm{RCP}}\left(X_{\mathrm{ION}}, \mathrm{TOF}\right)\right] /\right.$ $\left.\left[R_{\mathrm{RCP}}\left(X_{\mathrm{ION}}, \mathrm{TOF}\right)+S_{\mathrm{RCP}}\left(X_{\mathrm{ION}}, \mathrm{TOF}\right)\right]\right\}$ as a function of the ionic TOF and $X_{\mathrm{ION}} / \mathrm{mm}$ the position of impact onto the detector of the ion in the direction of light propagation. The count rate for each TOF and for each enantiomer and each helicity is normalized to one, whereby only the differential signal is represented; different integral ionization probabilities play no role in this representation. (d) Absolute ion count rate as a function of the ion TOF and position of impact on the ion detector. The transparent areas indicate the selection in (b). The vertical lines and their labels show the average TOF of the ions for the stated mass-to-charge ratios.

Figure 4 depicts that the fragments under considerationmay overlap in their flight times due to the momentum they received in the dissociation process. Thus, their masses cannot be unambiguously assigned. We therefore investigate the PICD as a function of the ion's time of flight. The dashed lines in Fig. 4 indicate the mean TOF of the fragments with $(m / q)=43,42,41,40,39$, and $38 \mathrm{amu} / \mathrm{au}$. While $(\mathrm{m} / \mathrm{q})=43$ indicates the singly charged ring fragment $\mathrm{C}_{2} \mathrm{H}_{3} \mathrm{O}$, the other mass-to-charge ratios can be reached via several fragmentation pathways. Different data recording times or imperfect circular polarizations directly influence the CD. Differences in the laser intensity can even change the weighting between the individual fragmentation channels [26]. It turns out that these sources of error largely compensate in the displayed difference of normalized differences [24]. A small constant offset was subtracted to compensate long-term laser drifts between the measurements of the two enantiomers (details in the Supplemental Material [5]). This demonstrates that for the CD in the ion yield a sensitive reference is needed. The CD signals presented in Fig. 4(a) are very small and in this case are not (much) larger than the experimental uncertainty. The statistical error is smaller than the estimated systematic errors. Therefore, instead of error bars we present a corresponding discussion in the Supplemental Material [5].

As before, the differential quantities show far stronger signals [Figs. 4(b) and 4(c)]. In Fig. 4(b), the same value as in Fig. 4(a) is displayed with the additional condition that the ionic fragment impinged the detector in or against the direction of light propagation [27].

The PICD as a function of the TOF and the position of impact on the ion detector is shown in Fig. 4(c). Before calculating the normalized difference, all four contributions from the two light helicities and enantiomers were normalized to 1 for each slice in the time of flight. As a result, the integral CD from the ion yield disappears and Fig. 4(c) only contains the influence of the $\mathrm{CD}$ in the differential ionization probability. Thus, some systematic errors in the measurement of the dichroism in the ion yield can be eliminated by the normalization. Figure 4(c) shows the PICD in this TOF region to be antisymmetric in forward and backward direction with a signal that is more than 5 times stronger than the CD signal [see Fig. 4(a)]. Interestingly, the strong dissociation channel for $(m / q)=$ 43 does not show a significant differential PICD. However, this finding does not have to mean that there is no differential PICD for this fragmentation channel for this fragment. Because of an imperfect correlation between the measured momentum vector of the ionic fragment and the molecular orientation, this observable might not be accessible in the present experiment. As confirmation of this hypothesis, no correlation between the measured ion momentum vector and the electron emission direction was found. Such a failure of the axial-recoil approximation can be caused by complex fragmentation dynamics.

Thus, the PICD in the single ionization of methyloxirane confirms an amplification of the signal, if a vectorial observable is examined instead of the integral ion yield. This increase is comparable to the presented case of the fourfold ionization of $\mathrm{CHBrClF}$. This trend suggests that here the dependence on the helicity of the light can influence the double differential ionization probability in a few percent range.

Our observations differ drastically from theoretical expectations in a weak-field limit. For ionization by a single photon, Cherepkov has shown that a possible enhancement of PICD for fixed-in-space chiral molecules is rather small [16]. This is because PICD in the one-photon ionization of oriented chiral molecules relies on the interferences between 
the electric-dipole with magnetic-dipole as well as electricdipole with electric-quadrupole interactions, and only the former interference survives averaging over molecular orientations and contributes to the conventional CD. Our experimental data demonstrate that the main strength of the differential PICD in the strong-field limit is provided by the electric-dipole interaction, with noticeable contributions from the aforementioned nondipole interactions. Explanation of the presently observed effect requires further development of the theory. Possible routes of development are discussed in the Supplemental Material [5].

In conclusion, we have shown that in the strong-field regime for a chiral molecule that is oriented in space the single and multiple ionization probability significantly depends on the helicity of the light. We have found changes in the ionization probability of up to $10 \%$. This is almost 2 orders of magnitude larger than what is typically found for randomly oriented molecules and is therefore comparable in terms of signal strength to the photoelectron circular dichroism. Our data show that the sign of the observed effect almost completely inverts upon mirroring of the geometry, which is the reason why the remaining effect, which survives averaging over all orientation of the fragments, is so small. From a technical perspective, our findings suggest that the enantioselectivity of ion detection can be much enhanced if in addition to the mass of the ion also one momentum component along the light propagation direction is detected. As we have shown, this is easily feasible by adding a position sensitive detector to a time-offlight mass spectrometer. Thus, we find that the influence of the light's helicity in strong-field ionization of chiral molecules differs drastically from the theoretical predictions for the case of single ionization by one photon. In addition, the differential PICD with the reported signal strength contributes significantly to the differential ionization probability in the strong-field regime, which should be considered in future theoretical models.

This work was funded by the Deutsche Forschungsgemeinschaft (DFG)-Project No. 328961117SFB 1319 ELCH (Extreme light for sensing and driving molecular chirality). K. F. and A. H. acknowledge support by the German National Merit Foundation. M. S. S. thanks the Adolf-Messer foundation and J.S. thanks Zürcher Hochschule für Angewandte Wissenschaften for financial support. Discussions with L. B. Madsen are gratefully acknowledged.

K. F., M. S. S., and R. D. declare no competing interests.

* Corresponding author.

fehre@atom.uni-frankfurt.de

Corresponding author.

schoeffler@atom.uni-frankfurt.de
Corresponding author.

doerner@atom.uni-frankfurt.de

[1] S. M. Kelly, T. J. Jess, and N. C. Price, Biochim. Biophys. Acta 1751, 119 (2005).

[2] F. S. Richardson and J. P. Riehl, Chem. Rev. 77, 773 (1977).

[3] E. A. Power and T. Thirunamachandran, J. Chem. Phys. 60, 3695 (1974); U. B. von Grafenstein and A. Bornschlegl, ChemPhysChem 7, 2085 (2006); C. S. Lehmann and K.-M. Weitzel, Phys. Chem. Chem. Phys. 22, 13707 (2020).

[4] H. G. Breunig, G. Urbasch, P. Horsch, J. Cordes, U. Koert, and K.-M. Weitzel, ChemPhysChem 10, 1199 (2009); P. Horsch, G. Urbasch, and K.-M. Weitzel, Chirality 24, 684 (2012).

[5] See Supplemental Material at http://link.aps.org/ supplemental/10.1103/PhysRevLett.126.083201 for (a) Discussion of systematic errors in Fig. 4. (b) Differences in the PICD when comparing the enantiomers or light helices. (c) Explanation on the PICD in single photon ionization. (d) Possible routes for the further development of the theoretical description., which include Refs. [6-8].

[6] A. D. Müller, E. Kutscher, A. N. Artemyev, and P. V. Demekhin, J. Chem. Phys. 152, 044302 (2020).

[7] M. Paul, L. Yue, and S. Gräfe, J. Mod. Opt. 64, 1104 (2017); S. Petretti, A. Saenz, A. Castro, and P. Decleva, Chem. Phys. 414, 45 (2013); S. Chelkowski, T. Bredtmann, and A.D. Bandrauk, Phys. Rev. A 85, 033404 (2012); A.-T. Le, R. R. Lucchese, S. Tonzani, T. Morishita, and C.D. Lin, Phys. Rev. A 80, 013401 (2009).

[8] S. Chattopadhyay, S. Bauch, and L. B. Madsen, Phys. Rev. A 92, 063423 (2015); S. Sukiasyan, S. Patchkovskii, O. Smirnova, T. Brabec, and M. Y. Ivanov, Phys. Rev. A 82, 043414 (2010); O. Smirnova, S. Patchkovskii, Y. Mairesse, N. Dudovich, D. Villeneuve, P. Corkum, and M. Y. Ivanov, Phys. Rev. Lett. 102, 063601 (2009); O. Smirnova, Y. Mairesse, S. Patchkovskii, N. Dudovich, D. Villeneuve, P. Corkum, and M. Y. Ivanov, Nature (London) 460, 972 (2009); S. Patchkovskii, Z. Zhao, T. Brabec, and D. M. Villeneuve, Phys. Rev. Lett. 97, 123003 (2006); A. Scrinzi, New J. Phys. 14, 085008 (2012); P. Wopperer, U. De Giovannini, and A. Rubio Eur. Phys. J. B 90, 51 (2017).

[9] A. F. Ordonez and O. Smirnova, Phys. Rev. A 98, 063428 (2018).

[10] B. Ritchie, Phys. Rev. A 13, 1411 (1976); N. Böwering, T. Lischke, B. Schmidtke, N. Müller, T. Khalil, and U. Heinzmann, Phys. Rev. Lett. 86, 1187 (2001); L. Nahon, G. A. Garcia, and I. Powis, J. Electron Spectrosc. Relat. Phenom. 204, 322 (2015); S. Turchini, N. Zema, G. Contini, G. Alberti, M. Alagia, S. Stranges, G. Fronzoni, M. Stener, P. Decleva, and T. Prosperi, Phys. Rev. A 70, 014502 (2004).

[11] C. Lux, M. Wollenhaupt, T. Bolze, Q. Liang, J. Köhler, C. Sarpe, and T. Baumert, Angew. Chem. Int. Ed. 51, 5001 (2012).

[12] S. Beaulieu et al., New J. Phys. 18, 102002 (2016).

[13] K. Fehre et al., J. Phys. Chem. A 123, 6491 (2019).

[14] I. V. Litvinyuk, K. F. Lee, P. W. Dooley, D. M. Rayner, D. M. Villeneuve, and P. B. Corkum, Phys. Rev. Lett. 90, 233003 (2003). 
[15] J. L. Hansen, L. Holmegaard, J. H. Nielsen, H. Stapelfeldt, D. Dimitrovski, and L. B. Madsen, J. Phys. B 45, 015101 (2012); A. I. Dnestryan, O. I. Tolstikhin, F. Jensen, and L. B. Madsen, Phys. Rev. Research 1, 023018 (2019).

[16] N. A. Cherepkov, Chem. Phys. Lett. 87, 344 (1982).

[17] K. Fehre et al., Sci. Adv. 5, eaau7923 (2019).

[18] R. Dörner, V. Mergel, O. Jagutzki, L. Sielberger, J. Ullrich, R. Moshammer, and H. Schmidt-Böcking, Phys. Rep. 330, 95 (2000); J. Ullrich, R. Moshammer, A. Dorn, R. Dörner, L. P. H. Schmidt, and H. Schmidt-Böcking, Rep. Prog. Phys. 66, 1463 (2003); T. Jahnke, T. Weber, T. Osipov, A. L. Landers, O. Jagutzki, L. P. H. Schmidt, C. L. Cocke, M. H. Prior, H. Schmidt-Böcking, and R. Dörner, J. Electron Spectrosc. Relat. Phenom. 141, 229 (2004).

[19] K. Fehre et al., Phys. Rev. Research 1, 033045 (2019).
[20] K. Fehre et al., Rev. Sci. Instrum. 89, 045112 (2018).

[21] E. J. McAlduff and K. N. Houk, Can. J. Chem. 55, 318 (1977).

[22] L. Arissian, C. Smeenk, F. Turner, C. Trallero, A. V. Sokolov, D. M. Villeneuve, A. Staudte, and P. B. Corkum, Phys. Rev. Lett. 105, 133002 (2010).

[23] M. Pitzer et al., Science 341, 1096 (2013).

[24] C. Lux, M. Wollenhaupt, C. Sarpe, and T. Baumert, ChemPhysChem 16, 115 (2015).

[25] R. M. Wood, Q. Zheng, A. K. Edwards, and M. A. Mangan, Rev. Sci. Instrum. 68, 1382 (1997).

[26] M. Murakami, R. Mizoguchi, Y. Shimada, T. Yatsuhashi, and N. Nakashima, Chem. Phys. Lett. 403, 238 (2005).

[27] A. Comby, E. Bloch, C. M. M. Bond, D. Descamps, J. Miles, S. Petit, S. Rozen, J. B. Greenwood, V. Blanchet, and Y. Mairesse, Nat. Commun. 9, 5212 (2018). 